\title{
Synthesis and characterizations of amorphous manganese oxide particles and continuous films for the application of rechargeable lithium batteries
}

\author{
Jafar F. Al-Sharab ${ }^{*}$ and Yiyun Yang ${ }^{1}$ \\ Department of Engineering Technology, Northwestern State University, Natchitoches, \\ LA 71497, USA \\ ${ }^{1}$ Formerly with the Department of Materials Science and Engineering, Rutgers \\ University, Piscataway, NJ 08854, USA
}

Manganese oxides have received increasing attention in the recent years due to its wide technological applications. The major applications of manganese oxides include advanced energy related materials such as batteries, catalysis applications, as well as other applications, such as water treatment, imaging contrast agents, and water oxidation $[1,2]$. The wide application of these oxides is due to its polymorphs, and crystalline structure. In the last decade, manganese oxide has been used as a high-capacity intercalation cathode for rechargeable lithium batteries. Most of the recent applications utilize nanoparticles and nanocomposites due to their high efficiency and unique properties. In this paper, we report a freeze-drying assisted synthesis of amorphous $\mathrm{MnO}_{2}$ material. The synthesized material was characterized thoroughly using various characterization techniques including TEM, SEM, XRD, and XPS. In addition, the synthesized material was evaluated as a host for lithium ions during charging-discharging process.

The fabrication of amorphous $\mathrm{MnO}_{2}$ was conducted using wet chemical route by mixing a calculated molar ratios of solid fumaric acid $\left(\mathrm{C}_{2} \mathrm{H}_{4} \mathrm{O}_{4}\right)$ and sodium permanganate $\left(\mathrm{NaMnO}_{4}\right)$, which produced a hydrogel. After rigorous rinsing in DI water, the unreacted materials and impurities in the hydrogels were freeze-dried under vacuum conditions for $\sim 12 \mathrm{hrs}$. This process yielded a porous and fluffy powder, Figure 1 (a). A high-resolution TEM micrograph of the synthesized $\mathrm{MnO}_{2}$ material is shown in Figure 1 (b). The inset in Figure 1(b) exhibits selected area electron diffraction of the imaged area. It is very obvious that the material lacks any crystallinity and can be referred as an amorphous. Additionally, the TEM micrograph shows that the material exhibits large, continuous, and wavy platelets. By observing the interaction of the electron beam with the amorphous particles, it is obvious that the platelets or films are most likely consist of few atomic layers. No evidence of crystallinity or beam damage was observed or found after beam exposure. The selected area electron diffraction (inset) consists of two major diffused rings, which correspond to short-range order at the atomic length. The dark bands observed in the HRTEM micrograph (Figure 1. (b)) correspond to the folding of the large film or an overlap of two or more films/particles.

The synthesized powder was evaluated as a lithium intercalation host. Voltage curves for charging-discharging were cycled between 3.3 to 1.5 Volt. The measured discharge specific capacity at rate of $0.06 \mathrm{~mA} / \mathrm{cm}^{2}$ was $290 \mathrm{mAh} / \mathrm{g}$. This value correspond to $\sim 1.06$ $\mathrm{Li} / \mathrm{Mn}$ which is a high value compared to many crystalline manganese oxides and other 
materials used in lithium ion batteries. Degradation in batteries is mainly related to cycling processes, often involving transformations among manganese oxide polymorphs, are governed by a complex interplay between thermodynamics and kinetics. In this paper the amorphous structure was evaluated as an alternate route to high-performance intercalation cathodes. Work is under way to test the electrochemical activity and further investigate the material for other technological applications. Furthermore, synthesis technique used here has a great potential to be applied to other nanostructured and nanocomposite materials.

\section{Acknowledgment:}

1) Paul F. Smith, Benjamin J. Deibert, Shivam Kaushik, Graeme Gardner, Shinjae Hwang, Hao Wang, Jafar F. Al-Sharab, Eric Garfunkel, Laura Fabris, Jing Li, and G. Charles Dismukes, "Coordination Geometry and Oxidation State Requirements of Corner-Sharing MnO6 Octahedra for Water Oxidation Catalysis: An Investigation of Manganite ( $\gamma$-MnOOH)" ACS Catal., 2016, 6 (3), pp 2089-2099, DOI: 10.1021/acscatal.6b00099

2) Chen, H, et. al; Facile Synthesis of Monodisperse Manganese Oxide Nanostructures and Their Applications in Water Treatment"; J. Phys. Chem., 112, 17540-17545, 2008

3) The TEM data were collected at the Electron Microscopy Facility at Rutgers University, Piscataway NJ, 08854

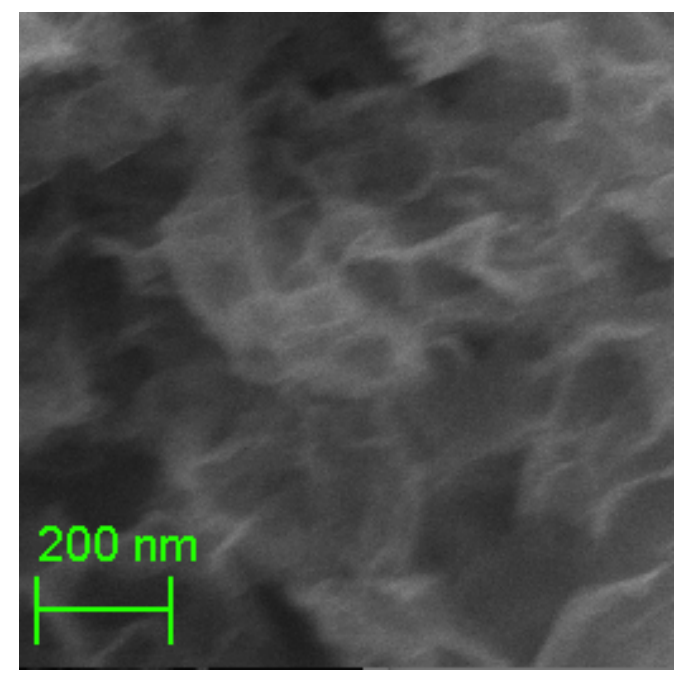

(a)

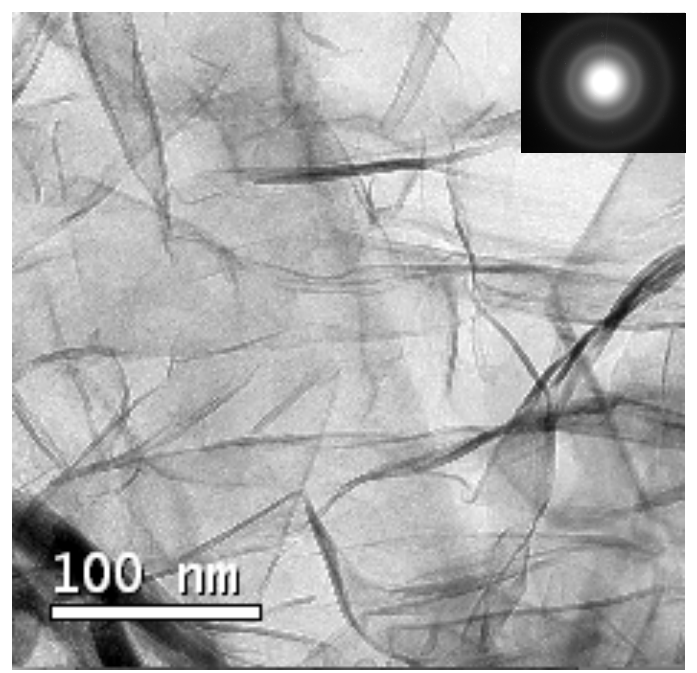

(b)

Figure 1. (a) Scanning Electron Micrograph of the as synthesized $\mathrm{MnO}_{2}$ powder. (b) High Resolution Transmission Electron Micrograph of the $\mathrm{MnO}_{2}$ particles. The inset in Figure 1(b) corresponds to selected area electron diffraction of the imaged area in (b). 